\title{
PERFIL CLÍNICO E NUTRICIONAL DE TRABALHADORES DE UMA UNIDADE HOSPITALAR CONFORME O TURNO DE TRABALHO
}

\section{Isadora Pinto Artuzo}

Nutricionista. Curso de Nutrição. Departamento de Educação Física e Saúde. Universidade de Santa Cruz do Sul (UNISC), Brasil.

\section{Fabiana Assmann Poll}

Nutricionista. Mestre em Ciências Médicas Pediatria (UFRGS). Docente do Curso de Nutrição. Departamento de Educação Física e Saúde. Universidade de Santa Cruz do Sul (UNISC), Brasil.

E-mail: fpoll@unisc.br

\section{Patricia Molz}

Nutricionista. Doutoranda em Medicina e Ciências da Saúde. Pontifícia Universidade Católica de Porto Alegre (PUCRS), Brasil.
RESUMO: Este artigo visa analisar os aspectos clínicos e nutricionais de trabalhadores de uma unidade hospitalar conforme o turno de trabalho. Foi realizado um estudo transversal com 40 trabalhadores diurnos e 40 noturnos. Analisou-se o consumo e frequência alimentar, classificou-se estado nutricional e risco cardiovascular e questionaramse aspectos do estilo de vida e condições de saúde. Constatou-se que a adequação do consumo de carboidratos e lipídeos foi similar e que as proteínas atingiram as recomendações de $100 \%$. O consumo de cálcio foi inadequado para $>90 \%$. Os trabalhadores noturnos apresentaram maior índice de massa corporal $(\mathrm{p}=0,006)$ e circunferência abdominal $(p=0,006)$ e mais dificuldades digestivas $(p=0,019)$. Observouse nos trabalhadores noturnos maior frequência de obesidade, maior risco cardiovascular e de alterações digestivas. O consumo alimentar de macronutrientes mostrou-se similar, o de cálcio foi inadequado e o leite, alimento-fonte, destacou-se por sua uma frequência de consumo ser de 1 a 3 vezes por semana.

PALAVRAS-CHAVE: Consumo de Alimentos; Estado Nutricional; Trabalho em Turnos.

\section{CLINICAL AND NUTRITIONAL PROFILE OF WORKERSOF A HOSPITAL ACCORDING TO THE WORK SHIFT}

\begin{abstract}
This paper aims to analyze the clinical and nutritional aspects of workers of a hospital according to the work shift. It was performed a cross-sectional study with 40 day workers and 40 night workers. The food consumption and frequency were analyzed, the nutritional status and cardiovascular risk were classified, and aspects of lifestyle and health conditions were questioned. It was found that adequacy of carbohydrate and lipid consumption was similar and proteins reached $100 \%$ of the recommendations. Calcium consumption was inadequate to $>90 \%$. Night workers had higher BMI $(p=0,006)$ and abdominal circumference $(p=0,006)$ and more digestive difficulties $(p=0,019)$. It was observed in the night workers more obesity frequency, higher cardiovascular risk and of digestive changes. The food consumption of macronutrients was similar, the calcium consumption was inadequate, and milk consumption, food source, stood out due to its consumptions frequency to be 1 to 3 times a week.
\end{abstract}

KEY WORDS: Food Consumption; Nutritional Status; Shift Work. 


\section{INTRODUÇÃO}

Rotinas exageradas de trabalho e turnos diferenciados podem causar alterações na alimentação. A realização de trabalho noturno também gera efeitos na saúde dos trabalhadores, tais como alterações biológicas no sono, desatenção, mudança no estado de ânimo e na vida social e familiar (MANHÂES, 2009).

Os ritmos circadianos, como o ciclo sono-vigília e o modo como os indivíduos alimentam-se são regidos internamente durante 24 horas. Este ritmo é responsável por conservar a sincronia entre os sistemas fisiológicos, comportamentais, hormonais e os sistemas moleculares, encarregados por diversos processos fisiológicos. Quando este funcionamento sofre algum tipo de modificação, podem ocorrer alterações no ritmo circadiano, as quais envolvem questões como alimentação e saciedade (O'REARDON et al., 2004).

O sono no período diurno não tem a mesma qualidade que o noturno, e, portanto, pode resultar em uma menor capacidade de produção do trabalhador durante à noite devido ao déficit de sono concentrado (OLIVEIRA, 2005). Os ritmos biológicos podem ser alterados devido à troca dos horários das refeições, ocorrendo mudanças na atividade enzimática de hormônios plasmáticos, como glucagon e insulina, assim como de metabólitos, colesterol, nitrogênio, triglicerídeos e corpos cetônicos (CRISTOFOLETTI,2003).

No século em que vivemos, com um ritmo de vida mais acelerado, os horários de descanso e refeições são mais escassos, o que pode contribuir para uma alimentação inadequada, com vistas à maior praticidade. Assim, observa-se um aumento na ingestão de lanches rápidos com qualidade nutritiv precária. Dessa forma, deixam o indivíduo mais vulnerável ao aparecimento das doenças cardiovasculares (SIMONETTI; KOBAYASHI; BIANCHI, 2010).

Para tanto, este estudo justifica-se pela identificação das fragilidades dos trabalhadores noturnos e diurnos no que tange a aspectos nutricionais, a fim de possibilitar a elaboração de programas de saúde e assistência voltadas para esse público.

Sendo assim, o objetivo do estudo foi analisar os aspectos clínicos e nutricionais de trabalhadores de uma unidade hospitalar conforme o turno de trabalho.

\section{METODOLOGIA}

Trata-se de um estudo com delineamento transversal, de caráter descritivo e observacional, aprovado pelo Comitê de Ética da Universidade de Santa Cruz do Sul, sob Parecer $n^{0}$ 1.337.729. O processo de amostragem deu-se por conveniência, com a intenção de manter o mesmo número de indivíduos nos dois turnos. A coleta dos dados foi realizada durante os meses de fevereiro e março de 2016. Foram incluídos no estudo trabalhadores diurnos e noturnos do hospital com idade superior a 18 anos, de ambos os sexos, que assinaram o Termo de Consentimento Livre e Esclarecido e que tivessem turno de trabalho definido.

Para identificar o consumo alimentar diário, foi aplicado um recordatório alimentar de 24 horas (R24h) de um dia da semana (FISBERG et al., 2005), que posteriormente foi digitado e analisado no software Avanutri ${ }^{\circledR}$. Analisou-se a distribuição dos macronutrientes em relação às calorias consumidas e o perfil de consumo de gordura saturada, colesterol, fibras, sódio e cálcio. Esses nutrientes foram escolhidos por serem importantes para a saúde cardiovascular, óssea e circulatória. Para estimar o consumo de sódio, o sal de adição considerado no cálculo do R24h foi aquele já contido nas preparações cozidas disponíveis no software.

Avaliou-se a ingestão dos micronutrientes através das Dietary Reference Intakes (DRIs) para faixa etária e gênero. Utilizou-se o Estimated Average Requirement (EAR) para avaliar a ingestão de cálcio. Usou-se a Adequate Intake (AI) para analisar o consumo de fibras, a Tolerable Upper Intake Level (UL) para sódio. Para os macronutrientes, utilizou-se o Acceptable Macronutrient Distribution Ranges (AMDR) (IOM, 2002). Os valores foram analisados conforme sua adequação de consumo para sexo e idade. O colesterol e a gordura saturada foram analisados em conformidade com as recomendações de Santos et al (2013).

Ainda, verificou-se a frequência de consumo alimentar (QFA) através de um questionário contendo grupos de alimentos consumidos na frequência semanal, quinzenal e mensal, adaptado de Lima e Gonzalez (2013). Questionou-se o consumo de cereais, frutas, legumes, lanches rápidos, doces e sobremesas, leite e derivados, 
carnes, embutidos, bebida alcoólica, refrigerante, frituras.

O estado nutricional foi avaliado pelo índice de massa corporal (IMC) (OMS,1997), calculado a partir da razão entre o peso corporal $(\mathrm{kg})$ e a estatura ao quadrado $\left(\mathrm{m}^{2)}\right)$. A aferição ocorreu com o indivíduo vestindo roupas leves e sem calçados, em balança digital Plena ${ }^{\circledR}$ com capacidade total de $150 \mathrm{~kg}$. A estatura (m), foi verificada com estadiômetro da marca Cardiomed ${ }^{\circledR}$ com capacidade de $2 \mathrm{~m}$ e com o indivíduo em posição ereta para a verificação da medida.

O risco cardiovascular foi identificado pela circunferência abdominal (CA), conforme critérios da I Diretriz Brasileira de Diagnóstico e Tratamento da Síndrome Metabólica (SOCIEDADE BRASILEIRA DE CARDIOLOGIA, 2005) aferido com fita métrica da marca Cardiomed $^{\circledR}$, no ponto médio entre a última costela e a crista ilíaca. Os participantes também foram questionados quanto aos aspectos do estilo de vida e condições de saúde, quais sejam: prática de exercícios físicos, hábito intestinal, presença de doença crônica, alergia ou intolerâncias alimentares e dificuldade de digestão ou deglutição.

Os dados foram tabulados e analisados no software StatisticalPackage for Social Sciences (SPSS) v.20.0. As variáveis contínuas foram descritas através de medidas de tendência central (médias) e de dispersão (desvio-padrão ou percentis), conforme a distribuição dos dados. Já as variáveis qualitativas, em número e percentual. Todas as variáveis quantitativas foram testadas para homocedasticidade (teste de Bartlet) e normalidade (teste de Kolmogorov Smirnov). Os testes $t$ de Student ou Mann-Whitney foram utilizados para comparar grupos. $\mathrm{O}$ teste qui-quadrado foi utilizado para comparar variáveis qualitativas. O nível descritivo do teste considerado foi de $\mathrm{p}<0,05$.

\section{RESULTADOS}

Ao todo, participaram do estudo 80 trabalhadores, sendo 40 por turno, e $72,5 \%$ eram do sexo feminino. A idade média global foi de 33,21 $\pm 8,67$ anos, sendo que dos trabalhadores diurnos foi de 29,95 $\pm 8,42$ anos e dos noturnos foi $36,48 \pm 7,72$ anos. O tempo mé- dio de trabalho na instituição do total dos indivíduos foi de 5,45 $\pm 6,76$ anos, sendo que no turno do dia foi de 2,44 $\pm 2,74$ anos e no da noite foi $8,46 \pm 8,14$ anos. A comparação entre os sexos, bem como dos dados clínicos, do estilo de vida e dos dados nutricionais estão dispostos na Tabela 1 . As doenças crônicas citadas pelos indivíduos estudados foram asma, hipertensão arterial sistêmica (HAS), hipotireoidismo e hipercolesterolemia e as relacionadas à digestão e deglutição foram azia, gastrite, refluxo e hérnia de hiato.

Tabela 1. Características clínicas, nutricionais e do estilo de vida entre trabalhadores avaliados, conforme o turno de trabalho - Santa Cruz do Sul, 2017

(continua)

\begin{tabular}{|c|c|c|c|c|}
\hline & $\begin{array}{c}\text { Turno Dia } \\
(n=40)\end{array}$ & $\begin{array}{c}\text { Turno } \\
\text { Noite } \\
(n=40)\end{array}$ & $\begin{array}{l}\text { Total } \\
(\mathrm{n}=80)\end{array}$ & $\mathbf{P}$ \\
\hline & n (\%) & n (\%) & \multicolumn{2}{|l|}{ n (\%) } \\
\hline \multicolumn{5}{|l|}{ Sexo } \\
\hline Feminino & $29(50,0)$ & $29(50,0)$ & $58(100)$ & \multirow{2}{*}{0,599} \\
\hline Masculino & $11(50,0)$ & $11(50,0)$ & $22(100)$ & \\
\hline \multicolumn{4}{|c|}{$\begin{array}{l}\text { Prática de atividade } \\
\text { física }\end{array}$} & \multirow[t]{3}{*}{0,263} \\
\hline Sim & $22(56,4)$ & $17(43,6)$ & $39(100)$ & \\
\hline Não & $18(43,9)$ & $23(56,1)$ & $41(100)$ & \\
\hline \multicolumn{5}{|l|}{$\begin{array}{l}\text { Alergia ou } \\
\text { intolerância } \\
\text { alimentar }\end{array}$} \\
\hline $\begin{array}{l}\text { Sim } \\
\text { Não }\end{array}$ & $\begin{array}{l}5(83,3) \\
35(47,3)\end{array}$ & $\begin{array}{l}1(16,7) \\
39(52,7)\end{array}$ & $\begin{array}{l}6(100) \\
74(100)\end{array}$ & 0,090 \\
\hline
\end{tabular}

Doenças crônicas

\begin{tabular}{|l|l|l|l|l|}
\hline $\begin{array}{l}\text { Sim } \\
\text { Nime }\end{array}$ & $8(53,3)$ & $15(100)$ & 0,775 \\
Não & $33(50,8)$ & $32(49,2)$ & $65(100)$ & \\
\begin{tabular}{|l|l|l|l|l|}
\hline $\begin{array}{l}\text { Dificuldade de } \\
\text { deglutição/digestão }\end{array}$ & & & & \\
\hline Sim & $9(32,1)$ & $21(40,4)$ & $52(100)$ & $0,019^{*}$ \\
\hline Não & $31(59,6)$ & $19(67,9)$ & $28(100)$ & \\
\hline
\end{tabular}
\end{tabular}

Hábito intestinal

\begin{tabular}{|l|c|c|c|c|}
\hline Normal & $29(48,3)$ & $31(51,7)$ & $60(100)$ & \\
Constipado & $10(55,6)$ & $8(44,4)$ & $18(100)$ & 0,866 \\
Diarreia & $1(50,0)$ & $1(50,0)$ & $2(100)$ & \\
\hline
\end{tabular}

Índice de Massa

Corporal 


\begin{tabular}{|c|c|c|c|c|}
\hline & $\begin{array}{c}\text { Turno Dia } \\
(n=40)\end{array}$ & $\begin{array}{l}\text { Turno } \\
\text { Noite } \\
(n=40)\end{array}$ & $\begin{array}{c}\text { Total } \\
(\mathrm{n}=80)\end{array}$ & $\mathbf{P}$ \\
\hline Desnutrição & $4(80,0)$ & $1(20,0)$ & $5(100)$ & \multirow{4}{*}{0,174} \\
\hline Eutrofia & $20(54,1)$ & $17(47,6)$ & $37(100)$ & \\
\hline Sobrepeso & $11(52,4)$ & $10(45,9)$ & $21(100)$ & \\
\hline Obesidade & $5(29,4)$ & $12(70,6)$ & $17(100)$ & \\
\hline \multicolumn{5}{|l|}{$\begin{array}{l}\text { Circunferência } \\
\text { Abdominal }\end{array}$} \\
\hline Sem Risco & $24(66,7)$ & $12(33,3)$ & $36(100)$ & \\
\hline $\begin{array}{l}\text { Risco de } \\
\text { Complicações } \\
\text { metabólicas } \\
\text { aumentado }\end{array}$ & $8(38,1)$ & $13(61,9)$ & $21(100)$ & $0,026 *$ \\
\hline $\begin{array}{l}\text { Risco de } \\
\text { Complicações } \\
\text { metabólicas } \\
\text { aumentado } \\
\text { substancialmente }\end{array}$ & $8(34,8)$ & $15(65,2)$ & $23(100)$ & \\
\hline
\end{tabular}

$* \mathrm{p}<0,05$. Teste quiquadrado.

A circunferência abdominal $(27,80 ; \mathrm{dp}=6,47 v$ s $24,26 ; \mathrm{dp}=4,62 ; \mathrm{p}=0,006)$ e o IMC $(89,35 ; \mathrm{dp}=14,12$ vs 81,$23 ; \mathrm{dp}=11,70 ; \mathrm{p}=0,006$ ) foram significativamente mais elevados no turno da noite (Figura 1).
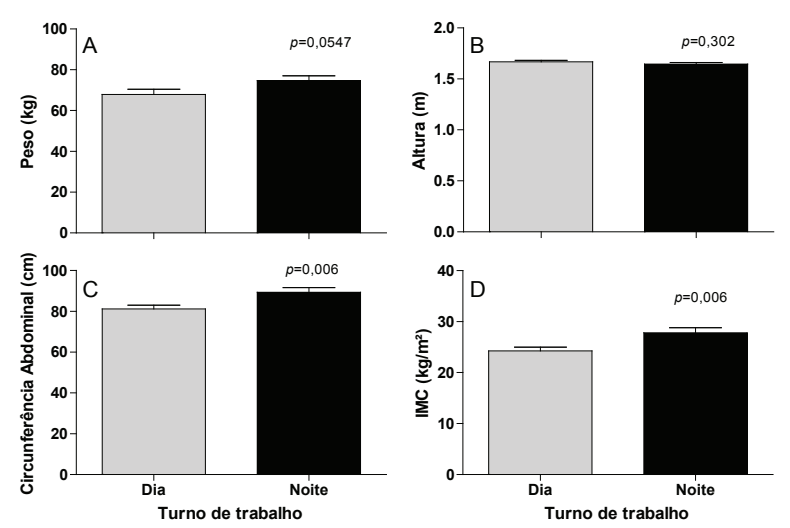

Figura 1. Comparação entre peso, altura, CA e IMC dos trabalhadores de cada turno (dia e noite), segundo o teste t de Student - Santa Cruz do Sul, 2017
Foi encontrada adequação de consumo de carboidratos entre 20 e $90 \%, 100 \%$ para proteínas e uma adequação de consumo entre 20 e $80 \%$ para os lipídeos de ambos os grupos de trabalhadores. A prevalência de consumo de gordura saturada não foi atingida por ambos os grupos. Quanto ao consumo do colesterol, 60\% dos trabalhadores noturnos e diurnos tiveram consumo abaixo da AI. O consumo de cálcio foi baixo, e a prevalência de inadequação foi maior do que $90 \%$ em ambos os grupos. O consumo de fibras abaixo da AI foi de $90 \%$ trabalhadores diurnos, porém entre 80 e $90 \%$ de inadequação no grupo noturno. A ingestão de sódio acima da UL foi de $60 \%$ e $70 \%$, para os trabalhadores diurnos e noturnos, respectivamente (Tabela 2).

Em relação à frequência alimentar, observou-se uma prevalência maior de consumo na frequência de $1 \mathrm{a}$ 3 vezes por semana, por ambos os turnos, dos seguintes grupos alimentares: embutidos, lanches, frituras, leite $\mathrm{e}$ derivados, bolo e cuca, doces e sobremesas e, por fim, refrigerantes e sucos industrializados (Tabela 3). 
Tabela 2. Avaliação da ingestão de nutrientes dos trabalhadores de acordo com o turno de trabalho - Santa Cruz do Sul, 2017

\begin{tabular}{|c|c|c|c|c|c|c|c|c|c|c|c|c|}
\hline \multicolumn{13}{|c|}{ TRABALHADORES DIURNOS } \\
\hline \multirow{2}{*}{ Nutrientes } & \multirow{2}{*}{$\begin{array}{l}\text { EAR AI } \\
\text { UL }\end{array}$} & \multirow{2}{*}{ Média(DP) } & \multicolumn{9}{|c|}{ Percentis } & \multirow{2}{*}{$\begin{array}{c}\text { Prevalência de ade- } \\
\text { quação }\end{array}$} \\
\hline & & & 10 & 20 & 30 & 40 & 50 & 60 & 70 & 80 & 90 & \\
\hline $\begin{array}{l}\text { Carboidratos (\%/ } \\
\text { dia) }\end{array}$ & $45-65$ & $53,70(10,13)$ & 37,83 & 44,46 & 50,53 & 52,23 & 56,59 & 58,63 & 59,75 & 62,62 & 66,06 & $\begin{array}{l}\text { Prevalência de adequação } \\
\text { é }>20 \% \text {, mas }<90 \%\end{array}$ \\
\hline $\begin{array}{l}\text { Proteínas (\%/ } \\
\text { dia) }\end{array}$ & $10-35$ & $17,85(4,93)$ & 11,53 & 13,71 & 14,69 & 16,18 & 17,10 & 18,58 & 19,96 & 22,66 & 25,94 & $\begin{array}{l}\text { Prevalência de adequação } \\
\text { é de } 100 \%\end{array}$ \\
\hline Lipídios (\%/dia) & $20-35$ & $28,45(7,81)$ & 19,74 & 21,87 & 22,66 & 25,31 & 28,13 & 30,25 & 32,17 & 35,58 & 40,56 & $\begin{array}{l}\text { Prevalência de adequação } \\
\text { é }>20 \% \text {, mas }<80 \%\end{array}$ \\
\hline Fibras (g/dia) & 25 & $12,98(5,12)$ & 6,49 & 7,60 & 9,03 & 10,22 & 12,75 & 15,08 & 16,80 & 17,18 & 20,49 & $\begin{array}{l}\text { Percentagem de ingestão } \\
\text { abaixo da } \mathrm{AI} \text { é > } 90 \%\end{array}$ \\
\hline $\begin{array}{l}\text { Gorduras satura- } \\
\text { das }(\% / \text { dia) }\end{array}$ & $<7 \%$ & $0,95(0,67)$ & 0,36 & 0,48 & 0,61 & 0,67 & 0,76 & 0,95 & 1,19 & 1,36 & 1,57 & $\begin{array}{l}\text { Percentagem de ingestão } \\
\text { abaixo da } \mathrm{AI}>90 \%\end{array}$ \\
\hline $\begin{array}{l}\text { Colesterol (mg/ } \\
\text { dia) }\end{array}$ & 200 & $214,88(153,93)$ & 55,21 & 79,68 & 110,85 & 160,24 & 196,50 & 227,06 & 262,48 & 303,36 & 401,92 & $\begin{array}{l}\text { Percentagem de ingestão } \\
\text { abaixo da } \mathrm{AI}>60 \%\end{array}$ \\
\hline Cálcio (mg/dia) & 800 & $392,57(328,32)$ & 97,22 & 135,10 & 176,21 & 235,66 & 274,05 & 404,04 & 489,87 & 575,74 & 757,21 & $\begin{array}{l}\text { Prevalência de inadequa- } \\
\text { ção é }>90 \%\end{array}$ \\
\hline Sódio (g/dia) & 2,3 & $2,01(1,20)$ & 0,67 & 1,02 & 1,33 & 1,51 & 1,76 & 2,20 & 2,54 & 2,93 & 3,10 & $\begin{array}{l}\text { Percentagem de ingestão } \\
\text { acima da } \mathrm{AI} \text { é }>60 \%\end{array}$ \\
\hline \multicolumn{13}{|c|}{ TRABALHADORES NOTURNOS } \\
\hline \multirow{2}{*}{ Nutrientes } & \multirow{2}{*}{$\begin{array}{l}\text { AMDR } \\
\text { EAR UL }\end{array}$} & \multirow{2}{*}{ Média DP } & \multicolumn{9}{|c|}{ Percentis } & \\
\hline & & & 10 & 20 & 30 & 40 & 50 & 60 & 70 & 80 & 90 & \\
\hline $\begin{array}{l}\text { Carboidratos (\%/ } \\
\text { dia) }\end{array}$ & $45-65$ & $54,34(10,85)$ & 39,05 & 44,63 & 48,04 & 53,08 & 55,62 & 57,83 & 60,13 & 62,08 & 70,02 & $\begin{array}{l}\text { Prevalência de adequação } \\
\text { é }>20 \% \text {, mas }<90 \%\end{array}$ \\
\hline $\begin{array}{l}\text { Proteínas (\%/ } \\
\text { dia) }\end{array}$ & $10-35$ & $16,60(5,11)$ & 10,62 & 12,80 & 13,78 & 14,77 & 16,05 & 17,09 & 17,41 & 19,63 & 23,32 & $\begin{array}{l}\text { Prevalência de adequação } \\
\text { é de } 100 \%\end{array}$ \\
\hline Lipídios (\%/dia) & $20-35$ & $29,17(9,01)$ & 18,61 & 22,45 & 24,05 & 26,73 & 28,75 & 30,91 & 33,50 & 36,06 & 39,15 & $\begin{array}{l}\text { Prevalência de adequação } \\
\text { é }>20 \% \text {, mas }<80 \%\end{array}$ \\
\hline Fibras (g/dia) & 25 & $11,73(7,31)$ & 5,01 & 6,10 & 6,92 & 7,96 & 9,30 & 10,46 & 13,25 & 18,52 & 25,01 & $\begin{array}{l}\text { Percentagem de ingestão } \\
\text { abaixo da AI é }>80 \% \text {, } \\
\text { mas }<90 \%\end{array}$ \\
\hline $\begin{array}{l}\text { Gorduras satura- } \\
\text { das }(\% / \text { dia) }\end{array}$ & $<7 \%$ & $1,03(0,36)$ & 0,65 & 0,75 & 0,77 & 0,87 & 1,03 & 1,08 & 1,26 & 1,37 & 1,51 & $\begin{array}{l}\text { Percentagem de ingestão } \\
\text { abaixo da } \mathrm{AI}>90 \%\end{array}$ \\
\hline $\begin{array}{l}\text { Colesterol (mg/ } \\
\text { dia) }\end{array}$ & 200 & $239,13(235,20)$ & 34,03 & 78,10 & 104,26 & 117,14 & 148,30 & 190,40 & 235,61 & 376,72 & 603,05 & $\begin{array}{l}\text { Percentagem de ingestão } \\
\text { abaixo da } \mathrm{AI}>60 \%\end{array}$ \\
\hline Cálcio (mg/dia) & 800 & $339,69(268,51)$ & 65,39 & 139,64 & 169,40 & 205,48 & 244,00 & 317,96 & 449,88 & 508,52 & 727,61 & $\begin{array}{l}\text { Prevalência de inadequa- } \\
\text { ção é }>90 \%\end{array}$ \\
\hline Sódio (g/dia) & 2,3 & $1,81(1,60)$ & 0,66 & 0,83 & 0,97 & 1,13 & 1,22 & 1,62 & 2,07 & 2,78 & 3,48 & $\begin{array}{l}\text { Percentagem de ingestão } \\
\text { acima da AI é }>70 \%\end{array}$ \\
\hline
\end{tabular}


Tabela 3. Comparação entre frequência de consumo de alimentos entre os trabalhadores da instituição, conforme o turno de trabalho - Santa Cruz do Sul, 2017

(continua)

\begin{tabular}{|c|c|c|c|c|}
\hline & Turno Dia $(n=40)$ & Turno Noite $(n=40)$ & Total (\%) & p \\
\hline \multicolumn{5}{|c|}{ n (\%) } \\
\hline Legumes & & & & \\
\hline Nunca & $1(33,3)$ & $2(66,7)$ & $3(100,0)$ & \multirow{5}{*}{0,596} \\
\hline Mensal/Quinzenal & $1(50,0)$ & $1(50,0)$ & $2(100,0)$ & \\
\hline 1 a 3 vezes por semana & $12(46,2)$ & $14(53,8)$ & $26(100,0)$ & \\
\hline 4 a 6 vezes por semana & $12(66,7)$ & $6(33,3)$ & $18(100,0)$ & \\
\hline Diariamente & $14(45,2)$ & $17(54,8)$ & $31(100,0)$ & \\
\hline \multicolumn{5}{|l|}{ Frutas } \\
\hline Nunca & $1(50,0)$ & $1(50,0)$ & $2(100,0)$ & \multirow{5}{*}{0,034} \\
\hline Mensal/Quinzenal & $5(100,0)$ & $0(0,0)$ & $5(100,0)$ & \\
\hline 1 a 3 vezes por semana & $4(28,6)$ & $10(71,4)$ & $14(100,0)$ & \\
\hline $4 \mathrm{a} 6$ vezes por semana & $11(68,8)$ & $5(31,3)$ & $16(100,0)$ & \\
\hline Diariamente & $19(44,2)$ & $24(55,8)$ & $43(100,0)$ & \\
\hline \multicolumn{5}{|l|}{ Saladas } \\
\hline Nunca & $2(100,0)$ & $0(0,0)$ & $2(100,0)$ & \multirow{5}{*}{0,417} \\
\hline Mensal/Quinzenal & $3(75,0)$ & $1(25,0)$ & $4(100,0)$ & \\
\hline 1 a 3 vezes por semana & $6(46,2)$ & $13(53,8)$ & $13(100,0)$ & \\
\hline 4 a 6 vezes por semana & $7(58,3)$ & $5(41,7)$ & $12(100,0)$ & \\
\hline Diariamente & $22(44,9)$ & $27(55,1)$ & $49(100,0)$ & \\
\hline \multicolumn{5}{|l|}{ Arroz } \\
\hline Nunca & $0(0,0)$ & $0(0,0)$ & $0(0,0)$ & \multirow{5}{*}{0,655} \\
\hline Mensal/Quinzenal & $1(50,0)$ & $1(50,0)$ & $2(100,0)$ & \\
\hline 1 a 3 vezes por semana & $5(50,0)$ & $5(50,0)$ & $10(100,0)$ & \\
\hline 4 a 6 vezes por semana & $8(66,7)$ & $4(33,3)$ & $12(100,0)$ & \\
\hline Diariamente & $26(46,4)$ & $30(53,6)$ & $56(100,0)$ & \\
\hline \multicolumn{5}{|l|}{ Leite e derivados } \\
\hline Nunca & $1(20,0)$ & $4(80,0)$ & $5(100,0)$ & \multirow{5}{*}{0,053} \\
\hline Mensal/Quinzenal & $3(75,0)$ & $1(25,0)$ & $4(100,0)$ & \\
\hline 1 a 3 vezes por semana & $14(63,6)$ & $8(36,4)$ & $22(100,0)$ & \\
\hline 4 a 6 vezes por semana & $8(27,3)$ & $3(27,3)$ & $11(100,0)$ & \\
\hline Diariamente & $14(36,8)$ & $24(63,2)$ & $38(100,0)$ & \\
\hline \multicolumn{5}{|l|}{ Carnes } \\
\hline Nunca & $0(0,0)$ & $0(0,0)$ & $0(0,0)$ & \multirow{5}{*}{0,413} \\
\hline Mensal/Quinzenal & $1(100,0)$ & $0(0,0)$ & $1(100,0)$ & \\
\hline 1 a 3 vezes por semana & $1(20,0)$ & $4(80,0)$ & $5(100,0)$ & \\
\hline $4 \mathrm{a} 6$ vezes por semana & $6(50,0)$ & $6(50,0)$ & $12(100,0)$ & \\
\hline Diariamente & $32(51,6)$ & $30(48,4)$ & $62(100,0)$ & \\
\hline \multicolumn{5}{|l|}{ Paes e biscoitos } \\
\hline Nunca & $0(0,0)$ & $1(100,0)$ & $1(100,0)$ & \multirow{5}{*}{0,508} \\
\hline Mensal/Quinzenal & $2(100,0)$ & $0(0,0)$ & $2(100,0)$ & \\
\hline 1 a 3 vezes por semana & $9(52,9)$ & $8(47,1)$ & $17(100,0)$ & \\
\hline 4 a 6 vezes por semana & $7(43,8)$ & $9(56,3)$ & $16(100,0)$ & \\
\hline Diariamente & $22(50,0)$ & $22(50,0)$ & $44(100,0)$ & \\
\hline
\end{tabular}


(conclusão)

\begin{tabular}{|c|c|c|c|c|}
\hline & Turno Dia $(n=40)$ & Turno Noite $(n=40)$ & Total (\%) & $\mathbf{p}$ \\
\hline \multicolumn{5}{|l|}{ Bolo/cuca } \\
\hline Nunca & $2(25,0)$ & $6(75,0)$ & $8(100,0)$ & \multirow{5}{*}{0,226} \\
\hline Mensal/Quinzenal & $15(68,2)$ & $7(31,8)$ & $22(100,0)$ & \\
\hline 1 a 3 vezes por semana & $17(43,6)$ & $22(56,4)$ & $39(100,0)$ & \\
\hline 4 a 6 vezes por semana & $5(55,6)$ & $4(44,4)$ & $9(100,0)$ & \\
\hline Diariamente & $1(50,0)$ & $1(50,0)$ & $2(100,0)$ & \\
\hline \multicolumn{5}{|l|}{ Lanches } \\
\hline Nunca & $1(25,0)$ & $3(75,0)$ & $4(100,0)$ & \\
\hline Mensal/Quinzenal & $12(44,4)$ & $15(55,6)$ & $27(100,0)$ & \\
\hline
\end{tabular}

1 a 3 vezes por semana

$24(63,2)$

$14(36,8)$

$38(100,0)$

$4 \mathrm{a} 6$ vezes por semana

$1(16,7)$

$5(83,3)$

$6(100,0)$

Diariamente

$2(40,0)$

$3(60,0)$

Doces/sobremesa

Nunca

$0(0,0)$

1

$5(100,0)$

Mensal/Quinzenal

$9(64,3)$

$1 \mathrm{a} 3$ vezes por semana

$19(46,3)$

$2(100,0)$

$2(100,0)$

$4 \mathrm{a} 6$ vezes por semana

$5(50,0)$

$5(35,7)$

$14(100,0)$

Diariamente

$5(50,0 \%)$

$22(53,7)$

$41(100,0)$

Embutidos

Nunca

$1(33,3)$

$5(50,0)$

$10(100,0)$

Mensal/Quinzenal

$7(63,6)$

$5(50,0)$

$1 \mathrm{a} 3$ vezes por semana

4 a 6 vezes por semana

Diariamente

$18(47,4)$

1

$10(100,0)$

Refrigerante/suco industrializado

$4(50,0)$

$10(50,0)$

$2(66,7)$

$3(100,0)$

Mensal/Quinzenal

1 a 3 vezes por semana

$1(14,3)$

$4(36,4)$

$11(100,0)$

$38(100,0)$

4 a 6 vezes por semana

Diariamente

$6(75,0)$

$20(52,6)$

$8(100,0)$

$10(50,0)$

$20(100,0)$

Frituras

$16(51,6)$

1

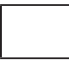

$6(85,7)$

$7(100,0)$

$$
\text { Nunca }
$$

$5(62,5)$

$2(25,0)$

$8(100,0)$

$15(48,4)$

$31(100,0)$

Mensal/Quinzenal

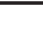

$12(46,2)$

$3(37,5)$

$8(100,0)$

$14(53,8)$

$26(100,0)$

1 a 3 vezes por semana

1

$4 \mathrm{a} 6$ vezes por semana

Diariamente

$1(33,3)$

$9(33,3)$

$2(66,7)$

$3(100,0)$

Álcool

Nunca

$26(68,4)$

$18(66,7)$

$27(100,0)$

$3(60,0)$

$12(31,6)$

$38(100,0)$

$1(14,3)$

$2(40,0)$

$6(85,7)$

$5(100,0)$

|

Mensal/Quinzenal

$6(28,6)$

$15(71,4)$

$7(100,0)$

1 a 3 vezes por semana

$15(53,6)$

$13(46,4)$

21(100,0)

4 a 6 vezes por semana

$17(63,0)$

$10(37,0)$

$28(100,0)$

$2(50,0)$

$2(50,0)$

$0(0,0)$

$0(0,0)$

*p $<0,05$. Teste qui-quadrado.

$4(100,0)$

$0(0,0)$

0,121




\section{DISCUSSÃO}

A presente pesquisa pretendeu identificar o perfil de trabalhadores diurnos e noturnos de um hospital no que diz respeito a sua alimentação, estado nutricional, aspectos clínicos e estilo de vida. De maneira geral, observou-se uma prevalência maior do sexo feminino nos maiores índices de sobrepeso e obesidade e risco cardiovascular em indivíduos que trabalham no turno da noite. Constatou-se uma adequação no consumo de carboidratos que variou entre 20 e $90 \%$ da recomendação, 20 e $80 \%$ de lipídeos e $100 \%$ de proteínas para ambos os grupos. O consumo de cálcio ficou abaixo do recomendado, com inadequação maior que $90 \%$ dos trabalhadores de ambos os grupos. O consumo de sódio acima da UL foi mais prevalente para os trabalhadores noturnos e observou-se um maior percentual do consumo de fibras abaixo da AI nesse mesmo grupo. O colesterol teve, para ambos os grupos, uma prevalência de consumo abaixo da AI de $60 \%$. Já o consumo de gordura saturada não atingiu o limite de recomendação em nenhum dos grupos (> P90). A frequência de ingestão diária de lanches, sucos e refrigerantes, frituras e frutas foi maior no turno da noite do que no turno do dia, apesar das diferenças não serem significativas.

Um estudo que comparou turnos de trabalho para avaliar a qualidade do sono e ansiedade de trabalhadores matutinos, vespertinos e noturnos em um hospital localizado em Campinas, com um total de 40 indivíduos, também verificou uma prevalência de $95 \%$ de mulheres (CAMPOS; DE MARTINO, 2004). Outro estudo similar realizado com operadores de telemarketing em duas centrais de atendimento telefônico que funcionavam em três turnos $(n=218)$, o qual visava avaliar o consumo alimentar e estado nutricional desses profissionais, a amostra também foi composta predominantemente pelo sexo feminino (72,5\%) e idade média de 28,9 9 7,6 anos (CRISTOFOLETTI, 2003). Entretanto, diferentemente do presente estudo, neste último somente 9,3\% dos trabalhadores foram classificados como obesos.

Foi realizada uma pesquisa com 94 trabalhadores noturnos de uma metalúrgica com a finalidade de conhecer seu estado nutricional e hábitos alimentares. Nele, $52,1 \%$ dos trabalhadores noturnos relataram consumir frequente ou muito frequente gorduras e frituras, $44,7 \%$ açúcares e doces, dados estes semelhantes ao presente estudo. Por outro lado, obtiveram o consumo de frutas e verduras e leite e derivados foi menos frequente, tendo sido relatado, respectivamente, por $27,7 \%$ e $43,6 \%$ dos trabalhadores (LOPEZ; SIMONY, 2013).

As dificuldades de digestão e deglutição foram citadas por trabalhadores de ambos os turnos, tendo prevalência maior nos trabalhadores do período da noite, constatação semelhante a do estudo de Lopez e Simony (2013), no qual foram identificados os mesmos sintomas, em sua maioria também nos trabalhadores noturnos (60,8\%) (LOPEZ; SIMONY, 2013).

Em relação ao consumo de cálcio, notou-se uma quantidade semelhante em ambos os turnos, porém abaixo das recomendações, por um grande número de trabalhadores. No QFA, o consumo de leite e derivados, em sua maior parte, foi relatado de 1 a 3 dias na semana, seguido de 4 a 6 vezes por semana e apenas por último o consumo diariamente, o que pode repercutir negativamente no teor de cálcio diário. Fato preocupante, pois como a amostra é composta em sua maioria por mulheres, isso pode ter repercussões futuras na saúde óssea. Após a menopausa, os ovários tornam-se inativos e ocorre mínima ou nenhuma liberação de estrogênio, coincidindo com a redução da absorção de cálcio pelo intestino devido à baixa produção de calcitonina, hormônio que inibe a desmineralização óssea (MISHRA et al., 2015).

O percentual de consumo de sódio dos trabalhadores noturnos acima da recomendação foi de $70 \%$ enquanto o consumo dos diurnos foi de $60 \%$ acima da UL. No entanto, o consumo de alimentos relatados no QFA que contém mais sódio foi maneira semelhante em ambos os turnos.

O risco cardiovascular dos trabalhadores do turno da noite foi maior. Contudo, quando somados os níveis de risco (aumentado e muito aumentado) do total da amostra e, principalmente, dos trabalhadores noturnos, verificou-se uma prevalência maior do que ausência de risco cardiovascular. Dessa forma, atenta-se ao perigo de uma circunferência abdominal aumentada para o desenvolvimento de complicações metabólicas, dentre elas a resistência à insulina. Esta pode ser transmitida geneticamente, mas há outro fator que aumenta essa chance, a 
obesidade abdominal, também conhecida por obesidade central, que leva à resistência insulínica e também acarreta em disfunções endoteliais (IANTTORNO et al., 2014; PRIETO; CONTRERAS; SÁNCHEZ, 2014). Assim, os trabalhadores noturnos, que podem sofrer mais com os efeitos de alterações no ritmo circadiano e suas consequências, aliados a um maior risco cardiovascular, índices de obesidade, e a menor prática de atividade física, teriam mais probabilidade de apresentar doenças crônicas não transmissíveis (FELLI, 2013).

Paralelo a isso, o percentual de ingestão de colesterol abaixo da AI foi semelhante em ambos os grupos (60\%). Santos et al (2013) recomendam uma ingestão diária de colesterol de até 300mg/dia. É importante ressaltar que a hipercolesterolemia é um dos principais fatores para a aterosclerose, podendo acarretar em acidente vascular encefálico e infarto do miocárdio (SANTOS et al, 2013).

A ingestão de gorduras saturadas foi observada em ambos os grupos de trabalhadores, mas com valores abaixo do percentual indicado de consumo, já que a recomendação é de até $7 \%$ do valor calórico total da dieta. O consumo de gordura saturada e trans é classicamente relacionado à elevação da lipoproteína de baixa densidade (LDL-c) plasmática e aumento de risco cardiovascular (SANTOS et al, 2013). Além disso, as consequências da ingestão de gordura não se limitam ao metabolismo lipídico, pois o tipo de gordura ingerida pode influenciar também outros fatores de risco, como a resistência à insulina e a pressão arterial (SANTOS et al, 2013).

Indivíduos que trabalham no ambiente hospitalar têm uma rotina que demanda muitas responsabilidades, atenção e acúmulo de tensão, que fazem parte dos cuidados a saúde de outras pessoas, que estão sob sua responsabilidade num momento frágil de suas vidas (KARINO et al., 2015). Consequentemente, hipotetizamos que essa rotina hospitalar acarrete em mudanças no estilo alimentar, levando a uma alimentação desbalanceada, muitas vezes associada à inatividade física, conduzindo ao excesso de peso corporal. Dessa forma, exige-se do indivíduo mais organização no seu cuidado pessoal e de saúde para evitar doenças futuras.

\section{CONCLUSÃO}

Ao final deste estudo pode-se concluir que os trabalhadores noturnos apresentaram maior IMC e risco cardiovascular e relataram mais dificuldades de digestão e deglutição. Constatou-se uma adequação de consumo de carboidratos que variou entre 20 e $90 \%$ da recomendação, entre 20 e $80 \%$ para lipídeos e 100\% para proteínaspara ambos os grupos. O consumo de cálcio ficou abaixo do recomendado, com inadequação maior que $90 \%$ para trabalhadores de ambos os grupos. O consumo de sódio acima da UL foi mais prevalente para os trabalhadores noturnos. Em relação a fibras, nesse mesmo grupo observou-se um maior percentual de consumo abaixo da AI. O colesterol teve, para ambos os grupos, uma prevalência de consumo abaixo da AI de $60 \%$, enquanto o consumo de gordura saturada não atingiu o limite de recomendação por nenhum dos grupos (>P90). A frequência de consumo de alimentos mais observada foi de 1 a 3 vezes na semana, tanto de alimentos que poderiam representar maior risco para a saúde, quanto os que apresentam benefícios. A prática de atividade física foi mais relatada pelos trabalhadores diurnos.

Constatou-se que ainda faltam estudos comparando esses mesmos fatores em turnos diferenciados para haver uma melhor comprovação das alterações acarretadas pela inversão do ritmo circadiano.

\section{REFERÊNCIAS}

ALLISON, K. C. et al. Cognitive behavior therapy for night eating syndrome: a pilot study. Association for the Advancement of Psychotherapy, Nova York, v. 64, n. 1, p. 91-106, 2010.

CAMPOS, M. L. P.; DE MARTINO, M. M. F. Aspectos cronobiológicos do ciclo vigília-sono e níveis de ansiedade dos enfermeiros nos diferentes turnos de trabalho. Revista da Escola de Enfermagem da USP, São Paulo, v. 38, n. 4, p. 415-421, dez. 2004.

CRISTOFOLETTI, M. F. Avaliação do estado nutricional de operadores de telemarketing submetidos a três 
turnos fixos de trabalho. 2003. 114f. Dissertação (Mestrado em Saúde Ambiental) - Faculdade de Saúde Pública da Universidade de São Paulo, São Paulo, 2003.

FELLI, V. E. A. et al. Indicators to surveillance the health of nursing staff. In: CONFERENCE ON EPIDEMIOLOGY IN OCCUPATIONAL HEALTH, 23., Anais...Utrecht, Holanda, 2013 .

FISBERG, R. M. et al. Inquéritos alimentares: métodos e bases científicos. 1. ed. Barueri: Manole, 2005.

IANTORNO, M. et al. Obesity, inflammation and endothelial dysfunction. J Biol Regul Homeost Agents., Birmingham, v. 28, n. 2, p. 169-176, abr. 2014.

INSTITUTE OF MEDICINE. Dietary Reference Intakes for Energy, Carbohydrate, Fiber, Fat, Fatty Acids, Cholesterol, Protein, and Amino Acids. Washington, D. C.: National Academy Press, 2002.

KARINO, M. E. et al. Cargas de trabalho e desgastes dos trabalhadores de enfermagem de um hospital-escola. Ciência, Cuidado e Saúde, Maringá, v. 14, n. 2, p. 10111018, jun. 2015.

LIMA, L. C.; GONZALES, M. C. Nutrição clínica no dia a dia. Rio de Janeiro: Rubio, 2013.

LOPEZ, J. E.; SIMONY, R. F. Hábitos alimentares e estado nutricional de trabalhadores noturnos de uma indústria metalúrgica da cidade de Guarulhos São Paulo, Brasil. Rev. Simbio- Logias, São Paulo, v. 6, n. 9, p. 117-129, dez. 2013.

MANHÃES, V. M. S. Cronotipo e privação do sono nos trabalhadores do serviço noturno hospitalar de enfermagem. 2009. 72f. Dissertação (Programa de Pós Graduação em Enfermagem da Universidade do Estado do Rio de Janeiro) - Universidade Federal do Rio de Janeiro, Rio de Janeiro, 2009.

MISHRA, S. et al. Comparison of bone mineral density and serum minerals in pre and post-menopausal women. International Journal of Clinical Trials, v. 2, n. 4, p. 85-90, nov. 2015.
O'REARDON, J. P. et al. Circadian eating and sleeping patterns in the night eating syndrome. Obes Res, Los Angeles, v. 12, n. 11, p. 1789-1796, nov. 2004.

OLIVEIRA, M. M. Alterações psicofisiológicas dos trabalhadores de enfermagem no serviço noturno. 2005. 130f. Dissertação (Programa de Pós graduação em Enfermagem, Escola de Enfermagem Anna Nery) - Universidade Estadual do Rio de Janeiro, Rio de Janeiro, 2005.

OMS. Organização Mundial de Saúde. Classificação estatística internacional de doenças e problemas relacionados a saúde. 5. ed. São Paulo: Editora da Universidade de São Paulo, 1997.

PRIETO, D.; CONTRERAS, C., SÁNCHEZ, A. Endothelial dysfunction, obesity and insulin resistance. Current vascular pharmacology, v. 12, n. 3, p. 412-426, mai. 2014.

SANTOS, R. D. et al. I Diretriz sobre o consumo de Gorduras e Saúde Cardiovascular. Arquivos Brasileiro de Cardiologia, São Paulo, v. 100, supl. 3, p. 1-40, 2013.

SIMONETTI, S. H.; KOBAYASHI, R. M.; BIANCHI, E. R. F. Identificação dos agravos à saúde do trabalhador de enfermagem em hospital cardiológico. Saúde Coletiva, Rio de Janeiro, v. 7, n. 41, p. 135-139, jan. 2010.

SBC. SOCIEDADE BRASILEIRA DE CARDIOLOGIA. I Diretriz Brasileira de Diagnóstico e Tratamento da Síndrome Metabólica. Arquivos Brasileiros de Cardiologia, São Paulo, v. 84, supl. 1, p. 1-28, abr. 2005.

SOCIEDADE BRASILEIRA DE CARDIOLOGIA; SOCIEDADE BRASILEIRADE HIPERTENSAO; SOCIEDADE BRASILEIRA DE NEFROLOGIA. VI Diretrizes Brasileiras de Hipertensão. Arquivos Brasileiros de Cardiologia, São Paulo, v. 95, n. 1, supl. 1, p. I-III, 2010.

Recebido em: 30 de naio de 2017 Aceito em: 30 de agosto de 2017 